\title{
Editors' Message: Eminent hydrogeologists profiled in 20 years of Hydrogeology Journal (1992-2012)
}

\author{
Sue M. Duncan • Clifford I. Voss
}

Keywords Profiles (of eminent hydrogeologists) · History of hydrogeology · Hydrogeology Journal

Since the early days of Hydrogeology Journal (HJ), when the journal was called Applied Hydrogeology, articles have been published that acknowledge the accomplishments of men and women who have been prominent in the science of hydrogeology. The intention has been to record the careers and work of eminent hydrogeologists, either of international renown or who have made significant contributions within their own counties. Their impact has been felt through development programs, through teaching, or whilst conducting research and hydrogeological studies that substantially enhance hydrogeologic science or the hydrogeology of a country (Wilson 1997).

Received: 16 May 2013 / Accepted: 31 May 2013

Published online: 25 July 2013

(C) Springer-Verlag Berlin Heidelberg 2013

S.M. Duncan is the technical editorial advisor for Hydrogeology Journal (HJ)

C.I. Voss is executive editor of $H J$

S. M. Duncan (

International Association of Hydrogeologists, PO Box 4130, Goring, Reading, RG8 6BJ, UK

e-mail: sduncan@iah.org

C. I. Voss

US Geological Survey,

345 Middlefield Road, MS 496, Menlo Park, CA 94025, USA

e-mail: cvoss@usgs.gov
To be considered as a subject, the career of the eminent hydrogeologist must be essentially completed due to retirement from active participation in the profession, or the hydrogeologist is deceased. Articles focus on the hydrogeologic activities and accomplishments of these people and on the impact they have had on colleagues and on the science. Thus, history has been recorded in $H J$ through descriptions of the careers of those who developed our science, in countries across the globe, from the 1730 s to the present day.

Since the start of the journal's publication in 1992, 32 such articles have been published (to end of 2012). The majority are in the article category "Profiles of Eminent Hydrogeologists" and several have been the subject of editorial messages. In addition, for some who have been profiled in $H J$ (and for some others), video recordings of interviews are freely available online via the International Association of Hydrogeologists (IAH) Hydrogeologist Time Capsule website (http://timecapsule.iah.org; Simmons and Renard 2008, 2010).

Table 1 lists the eminent hydrogeologists who have been profiled so far (1992 to 2012). These articles have all been unsolicited, contributed at the initiative of the authors. Eminent hydrogeologists are represented from Asia (India, China, Kazakhstan, Georgia, Iran, and Russia), North and South America (Argentina, Mexico, and USA), and Europe (Austria, France, Germany, Italy, The Netherlands and UK). Global coverage and sharing of hydrogeologic experience and innovation is an objective of these articles and there is no doubt that other hydrogeologists, unlisted as yet, deserve to be recorded in this series. The $H J$ editorial team would be pleased to receive nominations for eminent hydrogeologists and for potential authors of these Profiles (contact Clifford Voss, cvoss@usgs.gov). 
Table 1 Articles in Hydrogeology Journal that relate to eminent hydrogeologists (1992-2012). Arranged by continent

\begin{tabular}{|c|c|c|c|c|}
\hline Region $^{\mathrm{a}}$ & Eminent hydrogeologist ${ }^{\mathrm{b}}$ & Article title & Reference & Keywords $\left[{ }^{\mathrm{c}}\right]$ \\
\hline \multicolumn{5}{|l|}{ Europe } \\
\hline & $\begin{array}{l}\text { Joseph Elkington }(1739-1806), \\
\text { John Johnstone }(\sim 1796), \\
\text { John Wedge }(\sim 1794), \text { James } \\
\text { Anderson }(1739-1808), \\
\text { Francis Russell }(1765-1802), \\
\text { John Farey }(1766-1826), \\
\text { William Smith }(1769-1839)\end{array}$ & $\begin{array}{l}\text { British land drainers: their } \\
\text { place among pre-Darcy } \\
\text { forefathers of applied } \\
\text { hydrogeology }\end{array}$ & $\begin{array}{l}\text { Stephens and } \\
\text { Stephens } 2006\end{array}$ & $\begin{array}{l}\text { Profiles, applied } \\
\text { hydrogeology, conceptual } \\
\text { models, agriculture, } \\
\text { Great Britain, [UK] }\end{array}$ \\
\hline & $\begin{array}{l}\text { Baron Friedrich H.A. von } \\
\text { Humboldt (1769-1859), } \\
\text { Dominique-François J. } \\
\text { Arago (1786-1853) }\end{array}$ & $\begin{array}{l}\text { Humboldt, Arago, and the } \\
\text { temperature of groundwater }\end{array}$ & Davis 1999 & $\begin{array}{l}\text { Profiles, groundwater } \\
\text { temperature [France, } \\
\text { Germany] }\end{array}$ \\
\hline & \multirow[t]{4}{*}{ Henry Darcy (1803-1858) } & $\begin{array}{l}\text { Profiles of eminent } \\
\text { hydrogeologists: Henry } \\
\text { Darcy } 1803-1858\end{array}$ & Freeze 1994 & [France, Darcy] \\
\hline & & $\begin{array}{l}\text { Happy 200th birthday } \mathrm{Mr} \\
\text { Darcy and our thanks for } \\
\text { your law! A tribute editorial } \\
\text { celebrating the life and } \\
\text { times of the father of our } \\
\text { science, Henry Darcy } \\
(1803-1858)\end{array}$ & Simmons 2003 & [France, Darcy] \\
\hline & & Henry Darcy in his own words & Bobeck 2006 & $\begin{array}{l}\text { Profile, Darcy, Dijon, } \\
\text { France, water supply }\end{array}$ \\
\hline & & $\begin{array}{l}\text { Henry Darcy (1803-1858): } \\
\text { Immortalised by his } \\
\text { scientific legacy }\end{array}$ & Simmons 2008 & $\begin{array}{l}\text { Profiles, history of } \\
\text { hydrogeology, Henry } \\
\text { Darcy, Darcy's Law, } \\
\text { France, Dijon }\end{array}$ \\
\hline & $\begin{array}{l}\text { Jean-Baptiste de Lamarck } \\
\text { (b. 1744), Joseph Lucas } \\
\text { (1846-1926) }\end{array}$ & $\begin{array}{l}\text { Joseph Lucas and the term } \\
\text { "hydrogeology" }\end{array}$ & Mather 2001 & $\begin{array}{l}\text { General hydrogeology, } \\
\text { profiles (of eminent } \\
\text { hydrogeologists), } \\
\text { history of hydrogeology, } \\
\text { [Britain/UK, France] }\end{array}$ \\
\hline & $\begin{array}{l}\text { Willem Badon Ghijben } \\
\text { (1845-1907), Johan M.K. } \\
\text { Pennink (1851-1936) }\end{array}$ & $\begin{array}{l}\text { Willem Badon Ghijben and } \\
\text { Johan M.K. Pennink: } \\
\text { pioneers of coastal-dune } \\
\text { hydrology }\end{array}$ & de Vries 1994 & [The Netherlands] \\
\hline & Konrad Keilhack (1858-1944) & $\begin{array}{l}\text { Konrad Keilhack: Pioneer of } \\
\text { German Hydrogeology }\end{array}$ & Loehnert 1997 & [Germany] \\
\hline & Samuel Mandel (1918-1995) & $\begin{array}{l}\text { Samuel Mandel: International } \\
\text { Teacher of Hydrogeology }\end{array}$ & Limaye 1997 & [Austria] \\
\hline & Werner Käss (b. 1924) & $\begin{array}{l}\text { A conversation with Werner } \\
\text { Käss (Germany) about his } \\
\text { contributions to tracer } \\
\text { hydrogeology and } \\
\text { characterisation of mineral } \\
\text { waters and spas }\end{array}$ & $\begin{array}{l}\text { Goldscheider and } \\
\text { Goeppert } 2009\end{array}$ & $\begin{array}{l}\text { Profiles, interview, tracer } \\
\text { tests, thermal and mineral } \\
\text { water, Germany }\end{array}$ \\
\hline & $\begin{array}{l}\text { Costantino Faillace } \\
\text { (b. 1925) }\end{array}$ & $\begin{array}{l}\text { Costantino Faillace: } \\
\text { Philanthropic hydrogeologist }\end{array}$ & Limaye 1998 & $\begin{array}{l}\text { India, Italy, drinking } \\
\text { groundwater development, } \\
\text { water supply }\end{array}$ \\
\hline \multirow{5}{*}{ Asia } & $\begin{array}{l}\text { Nestor I. Tolstihin } \\
(1896-1992)\end{array}$ & $\begin{array}{l}\text { N.I. Tolstihin, a patriarch of } \\
\text { Russian hydrogeology }\end{array}$ & Zaltsberg 1995 & [Russia, permafrost] \\
\hline & $\begin{array}{l}\text { Gerasim Bogomolov } \\
\quad(1905-1981)\end{array}$ & $\begin{array}{l}\text { Academician G. Bogomolov } \\
(1905-1981)\end{array}$ & $\begin{array}{l}\text { Zaltsberg and } \\
\text { Chaban } 2006\end{array}$ & $\begin{array}{l}\text { Profiles, drilling, groundwater } \\
\text { resources, water supply, } \\
\text { Russia }\end{array}$ \\
\hline & Joseph Buachidze (b. 1906) & $\begin{array}{l}\text { Joseph Buachidze: Eminent } \\
\text { patriarch of the Georgian } \\
\text { hydrogeological community }\end{array}$ & $\begin{array}{l}\text { Zektser and } \\
\text { Varazashvili } 2000\end{array}$ & $\begin{array}{l}\text { Georgia, profiles, general } \\
\text { hydrogeology, groundwater } \\
\text { exploration, groundwater } \\
\text { development }\end{array}$ \\
\hline & $\begin{array}{l}\text { Daji Ganesh Limaye } \\
\text { (1909-1990) }\end{array}$ & $\begin{array}{l}\text { D. G. Limaye - a pioneer in } \\
\text { hard-rock hydrogeology in } \\
\text { India }\end{array}$ & Deolanker 1996 & [India] \\
\hline & $\begin{array}{l}\text { Boris I. Kudelin } \\
\quad(1910-1972)^{\mathrm{d}}\end{array}$ & $\begin{array}{l}\text { B.I. Kudelin: a pioneer of } \\
\text { regional evaluation of } \\
\text { groundwater resources } \\
\text { in Russia }\end{array}$ & Zaltsberg 2012 & $\begin{array}{l}\text { Profile, Russia, groundwater/ } \\
\text { surface-water relations, } \\
\text { groundwater resources, } \\
\text { groundwater mapping }\end{array}$ \\
\hline
\end{tabular}


Table 1 (continued)

\begin{tabular}{|c|c|c|c|c|}
\hline Region $^{\mathrm{a}}$ & Eminent hydrogeologist $^{\mathrm{b}}$ & Article title & Reference & Keywords [ $\left.{ }^{\mathrm{c}}\right]$ \\
\hline & $\begin{array}{l}\text { Alexei Konopliantsev } \\
\text { (b. 1912) }\end{array}$ & $\begin{array}{l}\text { Alexei Konopliantsev: A } \\
\text { doyen of the Russian } \\
\text { hydrogeological } \\
\text { community }\end{array}$ & Zaltsberg 1999 & $\begin{array}{l}\text { Russia, profiles, groundwater } \\
\text { monitoring, groundwater } \\
\text { statistics }\end{array}$ \\
\hline & $\begin{array}{l}\text { Ufa M. Akhmedsafin } \\
\text { (1912-1984) }\end{array}$ & $\begin{array}{l}\text { U.M. Akhmedsafin: a founder } \\
\text { of hydrogeological science } \\
\text { in Kazakhstan }\end{array}$ & Danilov-Danilyan 2012 & $\begin{array}{l}\text { Kazakhstan, profiles, } \\
\text { groundwater exploration, } \\
\text { hydrogeology of arid } \\
\text { regions, mapping }\end{array}$ \\
\hline & $\begin{array}{l}\text { Vladimir A. Kiryukhin } \\
\text { (b.1930) }\end{array}$ & $\begin{array}{l}\text { V.A. Kiryukhin, one of the } \\
\text { last champions of regional } \\
\text { hydrogeology (Russia) }\end{array}$ & Zaltsberg 2010 & Russia, profiles \\
\hline & Brij B.S. Singhal (b. 1931) & $\begin{array}{l}\text { BBS Singhal: a legend in } \\
\text { Indian hydrogeology }\end{array}$ & $\begin{array}{l}\text { Naik and Singhal } \\
2004\end{array}$ & $\begin{array}{l}\text { [India, geoscience, fractured } \\
\text { rocks] }\end{array}$ \\
\hline & Yuan Daoxian (b.1933) ${ }^{\mathrm{e}}$ & $\begin{array}{l}\text { Yuan Daoxian: an interview } \\
\text { with a distinguished } \\
\text { hydrogeologist from China }\end{array}$ & $\begin{array}{l}\text { Goldscheider et } \\
\text { al } 2008\end{array}$ & Profiles, interview, China \\
\hline & Ezatollah Raeisi (b. 1944) & $\begin{array}{l}\text { Ezatollah Raeisi: Iran's } \\
\text { foremost professor of } \\
\text { hydrogeology }\end{array}$ & Kazemi 2011 & Profiles, Iran \\
\hline & $\begin{array}{l}\text { Oktaviy Lange }(\sim 1950), \\
\text { Boris I. Kudelin } \\
\text { (1910-1972), Vsevolod } \\
\text { Shestakov }(\sim 1960), \text { Vladimir } \\
\text { Vsevolozhsky }(\sim 1980)\end{array}$ & $\begin{array}{l}\text { Fifty years of Moscow } \\
\text { University's Department } \\
\text { of Hydrogeology }\end{array}$ & $\begin{array}{l}\text { Zaltsberg and } \\
\text { Gogolev } 2003\end{array}$ & [Russia] \\
\hline \multicolumn{5}{|c|}{ North America } \\
\hline & T.C. Chamberlin (1843-1928) & $\begin{array}{l}\text { T.C. Chamberlin, early } \\
\text { American hydrogeologist }\end{array}$ & Back 1996 & [USA $]$ \\
\hline & Oscar E. Meinzer (1876-1948) & $\begin{array}{l}\text { Oscar E. Meinzer - father } \\
\text { of modern groundwater } \\
\text { hydrology in the United } \\
\text { States }\end{array}$ & Meyer 1995 & [USA $]$ \\
\hline & $\begin{array}{l}\text { Charles Vernon Theis } \\
(1900-1987)^{\mathrm{e}}\end{array}$ & An interview with C.V. Theis & Bredehoeft 2008 & $\begin{array}{l}\text { Profiles, history of } \\
\text { hydrogeology, [USA] }\end{array}$ \\
\hline & $\begin{array}{l}\text { Eugene S. Simpson } \\
(1917-1995)\end{array}$ & $\begin{array}{l}\text { Editorial: Tribute to Eugene } \\
\text { S. Simpson }\end{array}$ & $\begin{array}{l}\text { Neuman and } \\
\text { Campana } 1998\end{array}$ & $\begin{array}{l}\text { Profiles, Hydrogeology } \\
\text { Journal, editorial, [USA] }\end{array}$ \\
\hline & Paul Witherspoon (b. 1919) ${ }^{\mathrm{e}}$ & $\begin{array}{l}\text { An interview with Paul } \\
\text { Witherspoon, distinguished } \\
\text { hydrogeologist from the } \\
\text { USA }\end{array}$ & $\begin{array}{l}\text { Freeze and Javandel } \\
2008\end{array}$ & $\begin{array}{l}\text { Profiles, interview, USA, } \\
\text { Sweden }\end{array}$ \\
\hline & $\begin{array}{l}\text { Robert N. Farvolden } \\
\quad(1928-1995)\end{array}$ & $\begin{array}{l}\text { Robert N. Farvolden } \\
\text {-Pioneering contributions } \\
\text { in Mexican hydrogeology }\end{array}$ & Cortes and Durazo 2000 & $\begin{array}{l}\text { Profiles, general } \\
\text { hydrogeology, Mexico }\end{array}$ \\
\hline & $\begin{array}{l}\text { William B. White (b. 1934) } \\
\text { Elizabeth L. White } \\
\text { (b. 1936) }^{\mathrm{e}}\end{array}$ & $\begin{array}{l}\text { William B. White and } \\
\text { Elizabeth L. White } \\
\text { (USA): their contributions } \\
\text { to karst hydrogeology } \\
\text { discussed in an interview }\end{array}$ & Goldscheider et al 2009 & $\begin{array}{l}\text { Profiles, interview, USA, } \\
\text { karst, caves }\end{array}$ \\
\hline \multicolumn{5}{|c|}{ South America } \\
\hline & $\begin{array}{l}\text { Eduardo Usunoff } \\
(1954 ?-2008)\end{array}$ & $\begin{array}{l}\text { Editors' message: Eduardo } \\
\text { Usunoff }\end{array}$ & $\begin{array}{l}\text { Rodríguez and } \\
\text { Vives } 2008\end{array}$ & $\begin{array}{l}\text { Hydrogeology Journal, } \\
\text { Argentina, profiles }\end{array}$ \\
\hline
\end{tabular}

${ }^{\text {a }}$ Region from which the eminent hydrogeologist originated

${ }^{\mathrm{b}}$ (Year of birth and death); b. = born; =approximate year/s of active career (birth/death not recorded).

${ }^{\mathrm{c}}$ Keywords in brackets are added keywords (not originally designated)

${ }^{\mathrm{d}}$ See also Zaltsberg and Gogolev (2003) article Fifty years of Moscow University's Department of Hydrogeology

${ }^{\mathrm{e}}$ See also The Hydrogeologist Time Capsule (Simmons and Renard 2008, 2010). Eminent hydrogeologists that have been the subject of an interview, but for which there is not yet a published 'profile' in Hydrogeology Journal, also include: Jacob Bear, Gedeon Dagan, Shlomo P. Neuman, and József Tóth

\section{References}

Back W (1996) T.C. Chamberlin, early American hydrogeologist. Hydrogeol J 4(3):94-95. doi:10.1007/s10040-996-0004-9
Bobeck P (2006) Henry Darcy in his own words. Hydrogeol J 14:998-1004. doi:10.1007/s10040-005-0013-0

Bredehoeft JD (2008) An interview with C.V. Theis. Hydrogeol J 16:5-9. doi:10.1007/s10040-007-0248-Z 
Cortes A, Durazo J (2000) Robert N. Farvolden: pioneering contributions in Mexican hydrogeology. Hydrogeol J 8:251253. doi:10.1007/PL00021537

Danilov-Danilyan VI (2012) U.M. Akhmedsafin: a founder of hydrogeological science in Kazakhstan. Hydrogeol J 20:813816. doi:10.1007/s10040-012-0849-z

Davis SN (1999) Humboldt, Arago, and the temperature of groundwater. Hydrogeol J 7:501-503. doi:10.1007/s100400050223

de Vries JJ (1994) Willem Badon Ghijben and Johan M.K. Pennink: pioneers of coastal-dune hydrology. Appl Hydrogeol 2(4):5557. doi:10.1007/s10040-994-0002-8

Deolanker SB (1996) D.G. Limaye: a pioneer in hard-rock hydrogeology in India. Hydrogeol J 4(2):89-90. doi:10.1007/s10040-996-0012-9

Freeze RA (1994) Profiles of eminent hydrogeologists: Henry Darcy 1803-1858. Appl Hydrogeol 2(1):53. doi:10.1007/ s10040-994-0007-3

Freeze RA, Javandel I (2008) An interview with Paul Witherspoon, distinguished hydrogeologist from the USA. Hydrogeol $\mathrm{J}$ 16:811-815. doi:10.1007/s10040-008-0308-z

Goldscheider N, Goeppert N (2009) A conversation with Werner Käss (Germany) about his contributions to tracer hydrogeology and characterisation of mineral waters and spas. Hydrogeol J 17:1543-1546. doi:10.1007/s10040-009-0482-7

Goldscheider N, Yuexia W, Baker P (2008) Yuan Daoxian: an interview with a distinguished hydrogeologist from China. Hydrogeol J 16:1455-1457. doi:10.1007/s10040-008-0328-8

Goldscheider N, Baker P, Yuexia W, Groves C (2009) William B. White and Elizabeth L. White (USA): their contributions to karst hydrogeology discussed in an interview. Hydrogeol J 17:261-263. doi:10.1007/s10040-008-0337-7

Kazemi GA (2011) Ezatollah Raeisi: Iran's foremost professor of hydrogeology. Hydroegol J 19:501-503. doi:10.1007/s10040-0100683-0

Limaye SD (1997) Samuel Mandel: international teacher of hydrogeology. Hydrogeol J 5(3):124. doi:10.1007/s10040-997-0002-6

Limaye SD (1998) Costantino Faillace: philanthropic hydrogeologist. Hydrogeol J 6:323-324. doi:10.1007/s100400050155

Loehnert EP (1997) Konrad Keilhack: pioneer of German hydrogeology. Hydrogeol J 5(3):125. doi:10.1007/s10040-997-0003-5

Mather J (2001) Joseph Lucas and the term "hydrogeology". Hydrogeol J 9:413-415. doi:10.1007/s100400100145

Meyer G (1995) Oscar E. Meinzer: father of modern groundwater hydrology in the United States. Hydrogeol J 3(2):76-78. doi:10.1007/s10040-995-0005-0
Naik PK, Singhal DC (2004) BBS Singhal: a legend in Indian hydrogeology. Hydrogeol J 12:476-477. doi:10.1007/s10040004-0346-0

Neuman SP, Campana ME (1998) Editorial: Tribute to Eugene S. Simpson. Hydrogeol J 6:1-2. doi:10.1007/s100400050128

Rodríguez L, Vives LS (2008) Editors' message: Eduardo Usunoff. Hydrogeol J 16:1237. doi:10.1007/s10040-008-0361-7

Simmons CT (2003) Happy 200th birthday Mr Darcy and our thanks for your law! A tribute editorial celebrating the life and times of the father of our science, Henry Darcy (1803-1858). Hydrogeol J 11:611-614. doi:10.1007/s10040-003-0301-5

Simmons CT (2008) Henry Darcy (1803-1858): immortalised by his scientific legacy. Hydrogeol J 16:1023-1038. doi:10.1007/ s10040-008-0304-3

Simmons CT, Renard P (2008) Editors' Message: the Hydrogeologist Time Capsule - archival video recordings of influential hydrogeologists. Hydrogeol J 16(1):1-3

Simmons CT, Renard P (2010) The Hydrogeologist Time Capsule. http://timecapsule.iah.org/. Accessed Dec 2012

Stephens DB, Stephens DA (2006) British land drainers: their place among pre-Darcy forefathers of applied hydrogeology. Hydrogeol J 14:1367-1376. doi:10.1007/s10040-006-0052-1

Wilson EW (1997) Editors' messages: "Profiles of Eminent Hydrogeologists". Hydrogeol J 5(2):2

Zaltsberg E (1995) N.I. Tolstihin, a patriarch of Russian hydrogeology. Hydrogeol J 3(3):75-76. doi:10.1007/s10040-995-0003-2

Zaltsberg E (1999) Alexei Konopliantsev: a doyen of the Russian hydrogeological community. Hydrogeol J 7:419-420. doi:10.1007/s100400050213

Zaltsberg E (2010) V.A. Kiryukhin, one of the last champions of regional hydrogeology (Russia). Hydrogeol J 18:1053-1056. doi:10.1007/s10040-010-0583-3

Zaltsberg E (2012) B.I. Kudelin: a pioneer of regional evaluation of groundwater resources in Russia. Hydrogeol J 20:201-203. doi:10.1007/s10040-011-0785-3

Zaltsberg E, Chaban M (2006) Academician G. Bogomolov (19051981). Hydrogeol J 14:1377-1379. doi:10.1007/s10040-0060046-Z

Zaltsberg E, Gogolev M (2003) Fifty years of Moscow University's Department of Hydrogeology. Hydrogeol J 11:701-705. doi:10.1007/s10040-003-0304-2

Zektser I, Varazashvili L (2000) Joseph Buachidze: eminent patriarch of the Georgian hydrogeological community. Hydrogeol J 8:346-347. doi:10.1007/s100400050020 